Revue d'histoire de l'Amérique française

\title{
Lettre de Louis-Joseph Papineau à M. J.-J. Girouard, 30 mai 1855
}

Volume 6, numéro 4, mars 1953

URI : https://id.erudit.org/iderudit/301554ar

DOI : https://doi.org/10.7202/301554ar

Aller au sommaire du numéro

Éditeur(s)

Institut d'histoire de l'Amérique française

ISSN

0035-2357 (imprimé)

1492-1383 (numérique)

Découvrir la revue

Citer ce document

(1953). Lettre de Louis-Joseph Papineau à M. J.-J. Girouard, 30 mai 1855. Revue d'histoire de l'Amérique française, 6(4), 564-568.

https://doi.org/10.7202/301554ar d'utilisation que vous pouvez consulter en ligne.

https://apropos.erudit.org/fr/usagers/politique-dutilisation/ 


\title{
DOCUMENTS INÉDITS*
}

\author{
L.-J. Papineau à M. J.-J. Girouard ${ }^{1}$
}

De Monte-Bello, 30 Mai 1855.

\section{Mon cher Monsieur Girouard,}

Je commence par vous souhaiter \& à Madame, à votre famille \& aux amis du voisinage, santé \& contentement; à vous prier d'excuser le délais écoulé depuis la réception de votre lettre du dix-huit présent mois; et à vous expliquer les causes de ce délais. J'attendais de jour en jour Amédée ${ }^{2}$ de Montréal, et lui attendait de jour en jour, que notre chemin de fer que nous désirons si fort, non le vôtre, que vous désirez si peu, recommençat à rouler comme l'automne dernier, de Carillon à Grenville. Les Directeurs à Montréal espéraient en venir à un accomodement avec les propriétaires limitrophes. Ils n'y ont pas reussi: et Amédée pour éviter la fatigue du longsault, plus grande que jamais par la désorganisation du roulage sur la voie publique les propriétaires ayant vendu (stages) voitures et chevaux, il a pris enfin la résolution de venir par chemin de fer par voie de Rouse's Point, Ogdensburg et Byetown, et de là ici par le bateau à Vapeur: 150 lieues, en place des Vingt cinq de la route directe Et il la trouve tellement préférable qu'après m'avoir donné quatre jours ici, il est reparti par la même voie avec ses sours pour Montréal. Si nous avions notre chemin de fer, nous serions en deux heures à St Benoit et nous voisinerions; nous serions en trois heurres à

* Nous nous proposons d'offrir à nos lecteurs, quelques pièces documentaires, sur Girouard et sur les événements de 1837-1838. Ces documents sont reproduits de copies originales et font partie des Archives de l'Institut d'Histoire de l'Amérique française. Ces lettres de Papineau à Girouard, des biographes les ont déja citées en partie, jamais, à notre connaissance, intégralement. L.G.

1. J.-J. Girouard (Jean-Joseph). Né à Québec, 11 novembre 1795. Notaire a 21 ans, vient s'établir à Saint-Benoît, comté des Deux-Montagnes. Député de ce comté de 1831 a 1838. Placé par le gouverneur Gosford sur la liste des proscrits en 1837. Capturé, puis emprisonné à Montréal le 26 décembre 1837. Libéré le 16 juillet 1838, lors de l'amnistie de Durham. Emprisonné de nouveau le 4 novembre 1838; relâché le 27 décembre 1838. Refuse d'entrer dans le premier ministère LaFontaine en 1842. Meurt le 18 septembre 1855. Caractère sans reproche. "Patriote", mais opposé à la manière violente. Homme universellement estimé (Voir Aegidius Fauteux, Patriotes de 1837-1838, Êdition des Dix, (Montréal 1950), 253-56).

2. Avec Lactance et Gustave, l'un des trois fils de L.-J. Papineau. 
Montréal et ne resterions pas dans notre impénétrable solitude, où nous nous roillillons et devenons à vue d'œil de parfaits Algonquins. Il y a toute apparence que nous serons devenus tels irremédiablement, avant que les canons Russes ${ }^{3}$ soient tous pris $\&$ fondus en rails, ce quil nous faudra attendre sans doute, avant que toute entreprise de chemin de fer qui n'aura pas a sa disposition le pillage de la Province comme le grand tronc, puisse se continuer. Vous voyez donc qu'à part ma loyauté toujours la même pour la Mère Patrie, je suis interressé à la défaite des Russes. J'attendois donc Amédée pour savoir ce qui avait été fait du monument que vous aviez fait déposer sur les restes de mon cher Père ${ }^{4}$, lorsqu'il les avait dernièrement fait relever. Mon intention avait été de me trouver à Montréal à cette occasion, mais les médecins consultés sur les précautions à prendre, conseillèrent de le faire de suite avant que les chaleurs ne rendissent cette opération beaucoup plus difficile, si comme il y avait lieu de le craindre du laps de tems écoulé depuis 1841, la corruption du corps et des cercueils, avait été très grande. Sur cet avis Amédée obtint de suite du Juge \& de Monseigneur le Coadjuteur de Montréal, l'autorisation de faire faire cette exhumation \& de la bonté de Monsieur le Supérieur la permission de déposer le Corps, renfermé avec ses premiers cercueils dans un troisième en zinc Soudé, pour qu'il n'émanât point de mauvaise odeur, dans les voutes de l'église paroissiale, jusqu'à ce qu'il puisse être apportée ici. Si le chemin de fer avait glissé sur ses rails, il aurait pû être apporté ici par les bateaux à vapeurs et les locomotives. Il a fallu renoncer à l'idée de l'apporter par les secousses du roulage. Alors Amédée a projetté l'apporter par un des petits bateaux à vapeurs de tonage, mais leurs propriêtaires lui ont dit que les travaux du Grand trone avaient excessivement gâté la navigation du St Laurent à Ste Anne; que deux de ces bateaux y avaient échoué et n'avaient été dégagés qu'après de longs délais et que deux de leurs barges y avaient été brisées; qu'en conséquence ils lui conseillaient d'attendre aux eaux moyennes, où ces dangers cesseront, pour venir avec ces restes vénérés, ce qu'il fera sous un mois à peu près.

Lorsque j'eus l'extrême douleur de perdre ici mon cher Gustave, ${ }^{5}$ expiré avec la plus entière resignation à l'ordre de la Providence, à l'âge de vingt et un ans, dans les bras de ses inconsolables parents, Amédée accouru pour partager nos souffrances et assister aux funérailles de ce cher enfant, me proposa de bâtir une chapelle non loin de ma demeure \& qui dût servir de tombeau de famille. Oui lui dis-je je dois le faire. Je prends racine ici. Je ne m'éloignerai probablement pas, du lieu où repose celui que j'aurais dû

3. L'on était en pleine guerre de Crimée.

4. Père de Louis-Joseph Papineau (1753-1841). Député du Bas-Canada de 1792 à 1804 et de 1809 à 1814 . Propriétaire de la seigneurie de la Petite Nation en 1801. Mort en 1841.

5. Fils de Louis-Joseph Papineau, rédacteur à l'Avenir, à dix-sept ans. Mort d vingt et un ans, le 17 décembre 1851 . 
devancer. J'y réunirai le corps de mon Père et bientôt après mes restes seront déposés auprès. Mon cher Père a vaincu tant d'obstacles, surmonté tant de fatigues, fait tant de dépenses pour commencer l'établissement de cette Seigneurie, qu'il est bien, que nous y reposions les uns après les autres, aux pieds d'un homme qui a tant fait pour son pays et pour sa famille. Que son souvenir nous soit une leçon qui nous profite et aux enfans de nos enfans. J'ai donc durant les deux été derniers travaillé à cette chapelle qui n'est pas considérable, mais tant d'autres constructions, plantations \& défrichemens se suivent en même tems, qu'avec des ressources moyennes, l'on ne peut aller qu'un peu plus lentement que l'on ne voudrait. Elle est presque terminée; d'un stile gothique très uni, un peu agreste; tel qu'il me parait bien convenir au local et à la destination. Nous ne pouvons arriver à la maison ni en sortir sans passer tout près de notre dernière \& permanente demeure, située dans un joli boccage qui s'embellira avec la croissance de sa jeune forêt et les plantations additionelles projettées; à trois arpens de la résidence transitoire et passagère, à cinq de l'église paroissiale dont vous connaissez la situation. L'hiver dernier, j'ai eu la douleur d'y ouvrir et fermer la première tombe, pour le premier de mes petits fils, bel enfant d'un an emporté par sa première maladie. Le pauvre Père l'a apporté en fevrier dernier, pour le deposer dans la chapelle dont le premier, il avait formé le projet et tracé le plan presque constemment suivi. La seconde s'est ouverte \& fermée sur le corps de mon Cher Gustave, ces jours derniers pendant qu'Amédée était avec moi. I a troisième est construite pour recevoir bientot les restes de mon cher Père. Mais elle est béante, \& j'y puis tomber avant qu'ils arrivent. Que la volonté de Dieu soit faite en tout \& partout. Je puis laisser la vie sans regrets. J'y ai éprouvé trop de pertes désolantes pour y tenir fortement. Les avertissemens se multiplient trop rapides, pour que je puisse être surpris. De 22 écoliers entrés en Rhétorique en 1802, je suis le seul survivant. Mon ami Mr Antoine Parant ci devant supérieur du Séminaire de Québec, étai le seul de cette classe sur le bras duquel je pusse m'appuyer. Il n'avait qu'un ans plus que moi. Mon ami \& parent de cœur et d'enfance, avec qui j'ai été élevé comme frère, Louis Viger ${ }^{6}$ n'ayant aussi qu'un an plus que moi, tombe et je l'apprends pendant que je vous écris. Je dois me hâter de faire le peu de bien que je pourrai, puis aller rejoindre tant et tant de personnes que j'ai tendrement chéries.

Si Dieu me prête vie jusque là, j'assisterai à la Bénédiction de cette Chapelle par mon Evêque Diocésain ${ }^{7}$; dans les premiers jours d'Août. Je l'ai prié de venir faire cette cérémonie et d'en fixer le tems, il m'a donné par sa réponse reçue tout récemment, Ceux des dix premiers jours d'Août

6. Louis-Michel Viger (1785-1855), appelé "le beau Viger", avocat, député de Chambly de 1830 a 1838; de Nicolet de 1842 a 1844; de Terrebonne, de 1848 a 1851; de Leinster de 1841 a 1854; receveur-général dans le ministère Baldwin-LaFontaine. Meurt en 1855.

7. Joseph-Eugène-Bruno Guigues, premier évêque catholique d'Ottawa. 
qui me conviendraient le mieux et je lui ecris aujourd'hui même pour le prier de déterminer plus particulièrement celui qui lui conviendra, pour que je puisse l'indiquer à ceux des Parents et amis qui y viendront. Vous assistiez le 167 bre 1821, avec ces bons Amis que nous n'avons cessé de regretter, Messieurs Felix ${ }^{8}$ \& Dumouchel ${ }^{9}$ à la Benediction de la première église paroissiale. Nous sommes bien peu de survivans de cette première réunion. Vous assisterez je vous en prie aveo Madame votre épouse, $\mathrm{Mr}^{10} \&$ Madame Dumouchelle Mr \& Madame Le Maire à notre seconde bénédiction. Nous étions alors tous jeunes, peu éprouvés, peu attristés, pleins d'entrain, d'avenir, de projets; que de mécomptes. Néanmoins c'est la condition que l'ordre de la providence a établi dans tous les tems pour tous les hommes. Soyons soumis. Au plaisir de nous voir réunis de nouveau, se mêleront des souvenirs \& des regrets pour tous ceux que nous avons perdus; mais avec les regrets se mêle le plaisir de parler de leurs mérites, de leur amabilité, de leur affection pour nous. Nous rajeunissons un peu en parlant du bon vieux tems, des bons vieux amis. J'attendais la fin de ma correspondance avec Monseigneur de Bye-town pour vous faire cette prière, que je vous fais aujourd'hui et que je n'ai encore adressée à personne de la famille. Me Dessaulles ${ }^{11}$ et mes frères $\&$ les frères $\&$ sœurs de mon épouse $\&$ des neveux et des nièces et ma bonne belle sœur Angelle, sont ceux que j'espère réünir pour cette occasion avec les amis de St Benoit. D'ici là le jour précis sera déterminé et je vous en préviendrai. Je savais que je devais à votre sincère amitié pour mon Père \& Pour toute sa famille le monument si judicieusement choisi d'un cube, symbole de la solidité, de son caractère, de ses principes, de sa persévérance à suivre les études \& les entreprises utiles qu'il a successivement commencées \& continuées tant qu'il a respiré. Je devais vous demander que la Pierre tumulaire suivit les restes chers \& vénérés auxquels vous l'aviez consacrée. Vous me prévenez, parce que le moment de la dédicace de la Chapelle ou ils seront portés et du service funèbre pour les âmes des parents décédés et en particulier de ceux qui y sont déposés, qui y sera fait, n'êtait pas fixe. Votre partiale amitié a étendu trop loin les justes éloges

8. L'abbé Maurice-Joseph Lamedèque-Félix (1773-1831), curé de Saint-Benoít de 1802 à sa mort.

9. Jean-Baptiste Dumouchel (1784-1844). Marchand a Saint-Benoit vers 1810. Y épouse la sceur du curé Félix. Beau-frère de J.-J. Girouard. Emprisonné pendant les troubles de 1837.

10. S'agirait-il de Camille Dumouchel, l'un des fils de Jean-Baptiste et de Victoire Félix? "Patriote" comme son père. Émprisonné en 1837. Libéré à l'amnistie. Décédé en février 1882. Un autre fils de J.-Baptiste Dumouchel, appelé Hercule, celui-là, lui aussi "patriote" emprisonné en 1837 et qui vécut à Saint-Benoît, était décédé le 21 février 1854.

11. Jean Dessaules, beau-frère de Louis-Joseph Papineau, avait épousé Rosalie Papineau. 
que vous donnez à la Mémoire, aux vertus publiques \& privées qu'il eut, mon Père, aux services qu'il a rendus à la nationalité, a la patrie, à tant et tant de ses concitoyens, en les étendant à la famille, qui a marché sur ses traces il est vrai, mais d'un pas moins sur et moins heureux que lui. Il paraitrait donc un peu outré que dans une chapelle de famille \& privée quelqu'un de ses descendans eut écrit, Laboribus an Sobole clarior ${ }^{12}$. Mais les A.P.P. est un premier et suffisant correctif. Ensuite le Sobole ne s'applique pas à sa seule postérité, mais à sa postérité Morale patriotique, éclairée, qui sont tous les disciples que son exemple, et ses leçons ont fait naitre à la vie politique, à la défense énergique des droits de son pays, tous ceux qui sont tombés, tous ceux qui ont souffert pour cette Sainte-cause, dont il a été le doyen; ceux qui font autant d'honneur au Canada \& au Notariat comme Mr Girouard, et autres Notaires en Grand nombre depuis ce bon Mr Gagnier le plus ancien de ses clercs, jusqu'à J. Trudeau \& André Papineau les derniers qu'il a formés, tous ceux que ses encouragemens \& ses conseils ont aidés à se distinguer à leur tour, sont inclus dans l'expression. Si j'avais été présent c'eut été à moi à remplir le pieux devoir, que je n'aurais pu remplir aussi parfaitement bien, comme vous l'avez fait, sans vos conseils, mais ils ne m'auraient pas fait défaut. J'accepte donc avec reconnaissance l'Offre que vous me faites et que j'étais à la veille de vous demander, que le monument fut inséparable du corps sur lequel il a été posé. Il est demeuré au Cimetierre sur la fosse vide du corps de mon Père mais dans laquelle sont les restes réduits en poudre de cinq enfans morts en bas âges. Voilà la vie de l'homme, ses amertumes; ses inscrutables mistères, plus de la moitié de ce qui nait d'hommes, dévorés par la mort, avant qu'ils soient parvenus à cinq ans! O Abimes!

Faire du bien \& beaucoup et durable comme vous avez su le faire, Mon cher Mr Girouard, par la noble fondation que vous venez de terminer, voila un monument qui consacre à toujour votre mémoire et y attache les prières et les bénédictions de tous ceux qui y seront instruits et soulagés. Adieu.

Votre ami bien sincère et reconnaissant.

L.J. Papineau

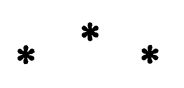

12. L'épitaphe au complet se lit comme suit:

\author{
JOSEPH PAPINEAU \\ Publicarum Legum Pater \\ Privatarum Expositor \\ Laboribus an sobole clarior \\ Obiit 8 Julii 1841 Olt 90 \\ Amici P.P.
}

